\title{
Estudo prospectivo e comparativo do escovado obtido pela CPER à ecoendoscopia associada à punção aspirativa com agulha fina (EE-PAAF) no diagnóstico diferencial das estenoses biliares
}

\section{Prospective comparative study of ERCP brush cytology and EUS-FNA for the diferential diagnosis of biliary strictures}

Monica Novis ${ }^{1}$; José Celso Ardengh ${ }^{1,2}$; Ermelindo Della Libera ${ }^{1}$; Frank Shigueo NakaO ${ }^{1}$; Laura Cota Ornellas ${ }^{1}$; Giulio Cesare Santo ${ }^{3}$; Filadelfio Venco ${ }^{3}$; Angelo Paulo Ferrari ${ }^{1}$

\section{RES U M O}

\begin{abstract}
Objetivo: Avaliar o desempenho diagnóstico da citologia obtida pela CPER, aquele obtido pela EE-PAAF e a concordância entre patologistas gerais $(\mathrm{PG})$ e especialistas (PE) em pacientes com estenose biliar. Métodos: Incluímos pacientes com estenose biliar identificados pela CPER. A EE-PAAF foi realizada apenas em áreas com efeito de massa ou da parede espessada do ducto biliar. O padrão-ouro foi a cirurgia, histologia e/ou o seguimento. As amostras teciduais foram consideradas: malignas, suspeitas, atípicas, insuficientes ou benignas. Os espécimes obtidos por cada método foi interpretado (cego) por um PG e outro PE. Resultado: 46 pacientes foram incluídos (37 malignos e 9 benignos). O diagnóstico final foi de tumor pancreático (26), biliar (11), pancreatite crônica (8) e estenose inflamatória do ducto biliar (1). Sensibilidade e acurácia da CPER foram 43,2\% e 52,2\% para o PG e $51,4 \%$ e $58,7 \%$ para o PE. Sensibilidade e acurácia da EE-PAAF foi $52,8 \%$ e $58,5 \%$ para o PG e $69,4 \%$ e $73,2 \%$ para o PE. A combinação entre a CPER e EE-PAAF demonstrou maior sensibilidade e acurácia para ambos PG $(64,9 \%$ e $69,6 \%)$ e PE (83,8\% e 84,8\%), respectivamente. Conclusão: A citologia obtida pelo escovado da via biliar durante a CPER e as amostras teciduais colhidas pela EE-PAAF tem rendimento semelhante para o diagnóstico das estenoses biliares. No entanto, a combinação dos métodos resulta em uma maior acurácia. Além disso, espera-se que a interpretação das amostras ocorra com maior precisão pelo PE se comparado ao PG.
\end{abstract}

Descritores: Ducto biliar/citologia. Neoplasma do trato biliar/patologia. Colangiopancreatografia endoscópica retrógrada. Colestase extrahepática/ patologia/ultrasonografia. Endosonografia/métodos.

\section{INTRODUÇÃO}

\begin{abstract}
A CPER geralmente é realizada para descomprimir a via biliar de pacientes com sintomas de obstrução maligna ${ }^{1}$. Muitos pacientes serão considerados inadequados para a cirurgia devido a doença localmente avançada ou a presença de metástases². Com os avanços da cirurgia pancreatobiliar e das técnicas de tratamento paliativo com próteses metálicas autoexpansíveis, o diagnóstico diferencial preciso de lesões benignas e malignas da via biliar se tornou essencial para o planejamento terapêutico adequa$\mathrm{do}^{3,4}$. Além disso, o tratamento por quimioterapia ou radioterapia requer diagnóstico histológico preciso'.

A técnica ideal para colher amostra tecidual durante a CPER deve ter alta sensibilidade e especificidade.
\end{abstract}

A técnica deve ser simples, rápida e segura, para que seja amplamente utilizada. Infelizmente, nenhum dos métodos atuais de coleta pela CPER possui essas características'.

Embora o escovado da estenose pela CPER continue a ser o método mais conveniente e utilizado para colher amostra tecidual, a quantidade insuficiente de células tumorais contribui para sensibilidade e valor preditivo negativo baixos ${ }^{5}$. Todos os métodos atuais apresentam sensibilidade que varia entre $20 \%$ e $60 \%$, com especificidade de $100 \%{ }^{1}$. Essa última, provavelmente é o resultado da rigorosa interpretação por parte dos patologistas dos espécimes, que diagnosticam malignidade somente nos casos óbvios ${ }^{6}$.

No entanto, a interpretação das amostras teciduais pode ser examinador dependente. Por exemplo,

Trabalho realizado na Universidade Federal de São Paulo - INIFESP.

1.Doutora pela UNIFESP, Unidade de Endoscopia, Divisão de Gastroenterologia, Universidade Federal de São Paulo, Brasil; 2. Livre Docente do Departamento de Cirurgia e Anatomia da Faculdade de Medicina de Ribeirão Preto, Universidade de São Paulo, Ribeirão Preto, São Paulo, Brasil; 3. Médico da Unidade de Diagnóstico Anatomopatológico - Universidade Federal de São Paulo - UNIFESP; 4. Professor adjunto da Disciplina de Gastroenterologia da UNIFESP. 
um bom citopatologista chega a um diagnóstico definitivo, mesmo que poucas células anormais sejam identificadas? Vários estudos anteriores que examinaram o papel da EEPAAF na abordagem das estenoses biliares mostraram sensibilidade que varia entre $43 \%$ e $86 \%$ em estenoses biliares e entre $25 \%$ e $83 \%$ na estenoses biliares proximais ${ }^{8-13}$. Entretanto, a maioria desses estudos é retrospectivo, apresenta amostragem limitada ou requer uma metodologia mais rígida.

O objetivo deste estudo foi avaliar prospectivamente a acurácia diagnóstica do escovado obtido pela CPER com os resultados obtidos pela EE-PAAF nas estenoses biliares. Outro ponto importante analisado foi a concordância entre patologistas gerais e gastrointestinais no diagnóstico etiológico final das estenoses biliares.

\section{MÉTODOS}

O protocolo do estudo foi aprovado pelo ConseIho de Ética em pesquisa da UNIFESP Hospital São Paulo número 0625/01.

Quarenta e seis pacientes atendidos consecutivamente, foram incluídos neste estudo prospectivo, realizado na Unidade de Endoscopia, Divisão de Gastrenterologia, Universidade Federal de São Paulo, Brasil. Todos eram portadores de obstrução biliar distal, local de onde foram colhidas amostras teciduais para diagnóstico e planejamento terapêutico. Os critérios de exclusão foram: tumor da papila duodenal, tumor invadindo o duodeno (adequado para biópsias endoscópicas regulares), suspeita clínica de obstrução benigna pós-operatória, diagnóstico prévio de malignidade através de biópsia, coagulopatia, impossibilidade de passar o fio-guia através da estenose, recusa em assinar o consentimento informado, comorbidades graves ou estado geral comprometido. Os pacientes que se perderam durante o "follow-up", aqueles com biópsia prévia negativa e com seguimento inferior a 24 meses também foram excluídos. Todos os pacientes foram submetidos à CPER e EE-PAAF realizadas por dois endoscopistas terapêuticos experientes, APF e JCA, respectivamente. $\mathrm{O}$ endoscopista experiente foi definido como aquele que realizou mais de 3000 CPER ou EE. Os procedimentos foram realizados em sessões separadas, com no máximo duas semanas de intervalo. Os endoscopistas foram randomizados para os resultados dos testes anteriores. Embora a seqüência dos exames não tenha sido padronizada, a CPER foi o primeiro procedimento a ser realizado em $91 \%$ dos casos. Outros exames de imagem (US, tomografia computadorizada, ressonância magnética) foram realizados conforme o caso.

A CPER foi realizada pela técnica padrão, utilizando-se um videoduodenoscópio terapêutico Pentax ED 3440 (Pentax Precision Instruments Corp, Orangeburg, Nova York). Após injeção de contraste (ioxitalamato de meglumina) na árvore biliar, o nível, extensão e o aspecto da estenose biliar foram avaliados. Após a passagem de um fio-guia, uma escova da Geenen $₫$ (Wilson-Cook Medical Inc., Winston-Salem, NC) foi usada para obter amostras citológicas sob controle radiológico (Figura 1). As estenoses foram escovadas várias vezes com movimentos repetidos de vai-e-vem. A escova foi retirada passando por dentro da sua bainha de revestimento. Os casos em que foram colhidas amostras do ducto pancreático principal (quatro pacientes) não foram computados nos resultados.

Quando indicado, o tratamento endoscópico, com dilatação da estenose e a colocação de prótese, foi
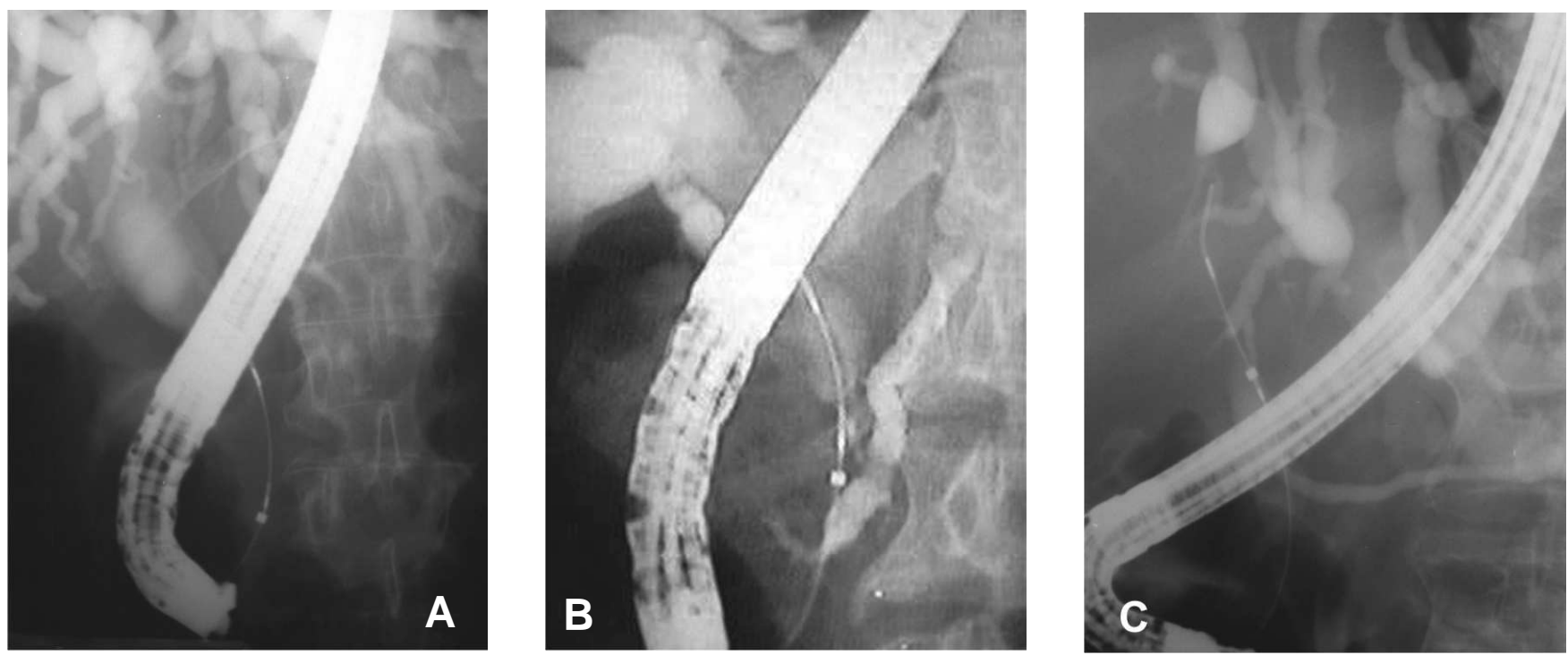

Figura 1 - A, B e C - Controle fluoroscópico do momento do uso do sistema de escova Geenen ${ }^{\circledR}$ da Wilson-Cook, para a obtenção de espécimes citológicos. A escova deve ser acionada com movimentos de vai-e-vem junto a estenose biliar para trumatizar a estenose e coletar células. 
realizado imediatamente após o escovado. O material celular aderido à escova foi semeado em seis lâminas de vidro, imediatamente fixados em etanol 95\% (três lâminas de vidro) e secos ao ar (três lâminas de vidro) na sala de endoscopia. A EUS foi realizada com um ecoendoscópio setorial Pentax FG 36-UX (Pentax Precision Instruments Corp, Orangeburg, Nova York) acoplado a uma unidade de ultrasom Hitachi EUB 505 (Mitsubishi, Conshockon, Filadélfia). A EE-PAAF foi realizada na presença de massa na cabeça do pâncreas ou ao redor do ducto biliar, ou em área focal de espessamento na parede do ducto biliar. Quando a massa não foi claramente demonstrada pela EE, a punção com agulha não foi tentada e a estenose foi classificada como benigna, com base apenas em seu aspecto ultrasonográfico. A EE-PAAF foi realizada utilizando uma agulha longa de 145 centímetros e 22 gauge GIP $®$ (Medi Globe GmbH Alemanha). Após penetrar na lesão o mandril era removido fazendo-se a sucção com seringa de $20 \mathrm{ml}$, enquanto se movia a agulha no interior da lesão (Figura 2). Pelo menos três passagens da agulha foram feitas em todos os casos. Antes de retirar a agulha a sucção foi interrompida ao desconectar a seringa. Após retirar a agulha, o aspirado foi coletado. Os espécimes de "core" biópsia foram fixados em formol e o conteúdo aspirado foi esfregado em seis lâminas de vidro e imediatamente fixado em etanol a 95\% (três lâminas de vidro) e secos ao ar (três lâminas de vidro). O material foi avaliado pelo ultrasonografista (JCA) para saber se era ou não adequado para diagnóstico, em particular quanto à presença de material visível e não apenas sangue. O material aspirado e os espécimes de core biópsias foram incluídos e analisados como amostra única.

Quando o paciente tinha sido submetido à procedimentos repetidos, apenas os resultados obtidos pelas primeiras CPER e EE foram registrados. O resultado positivo ou negativo obtido pelo follow-up com CPER ou EE, foi considerado apenas como uma referência para o diagnóstico etiológico final. Os citologistas que avaliaram os esfregaços obtidos pela CPER, bem como o material obtido com EE-PAAF não estavam presentes durante os procedimentos. Os espécimes citológicos foram corados pelo Papanicolau (etanol 95\% fixo) e Giemsa (fixados a seco). A análise histopatológica foi realizada após fixação em formol, inclusão em parafina, e imersão em corante $(H$ \& $\mathrm{E}$, e reativo de Schiff) do material em cortes de 5 mícrons e classificado segundo os critérios da Organização Mundial de Saúde ${ }^{14}$.

Todos os espécimes foram interpretados por patologistas gerais e GI (GCS e FV). Eles foram interpretados sem o conhecimento dos dados clínicos e endoscópicos, assim como dos resultados das amostras anteriores (Figura 3). Os espécimes foram classificados como positivo, suspeito e negativo para células malignas, atípico e número insuficiente de células ${ }^{15,16}$. O diagnóstico final baseou-se no exame do espécime cirúrgico e em autópsia, quando disponível. O resultado positivo com qualquer método de aquisição de tecido foi suficiente para diagnosticar malignidade, quando associado ao acompanhamento clínico, desde que houvesse sinais evidentes de deterioração clínica ou morte dentro de 6 meses a partir da linha de base. Na ausência de confirmação histológica, o diagnóstico de câncer foi feito quando havia evidência de malignidade através de imagem acrescida de seguimento clínico que sugeria doença maligna. Os pacientes que estavam vivos e estáveis e/ou apresentavam melhora com o tratamento cirúrgico ou endoscópico, durante pelo menos 24 meses foram considerados como portadores de doença benigna. Os dados foram coletados a partir da revisão de registros médicos e de contatos telefônicos com os pacientes, parentes, e seus respectivos clínicos e cirurgiões.

\section{Análise Estatística}

Um consentimento informado foi obtido de todos os pacientes. Para efeitos de análise estatística, as amostras com material insuficiente e negativa para malignidade foram classificadas como negativas, enquanto que os espécimes com células atípicas e suspeitas de malignidade foram agrupados com as amostras positivas para malignidade, e classificados como positivos. A sensibilidade, especificidade, valor preditivo positivo, valor preditivo negativo e acurácia diagnóstica foram calculados para cada tipo de amostra e combinação de métodos. Para avaliar o significado da atipia, esses parâmetros foram também calculados apenas para os casos positivos ou suspeitos considerados como equivalente ao câncer. Para avaliar o desempenho diagnóstico dos métodos combinados, o resultado foi considerado positivo quando, com qualquer um dos dois métodos, o resultado foi positivo para malignidade. Para a comparação dos dados quantitativos, o teste $T$ de Student foi utilizado. O teste do qui quadrado e o teste exato de Fisher foram utilizados para comparação das proporções. O coeficiente Kappa (k) foi utilizado para medir a concordância interobservador. A razão de verossimilhança

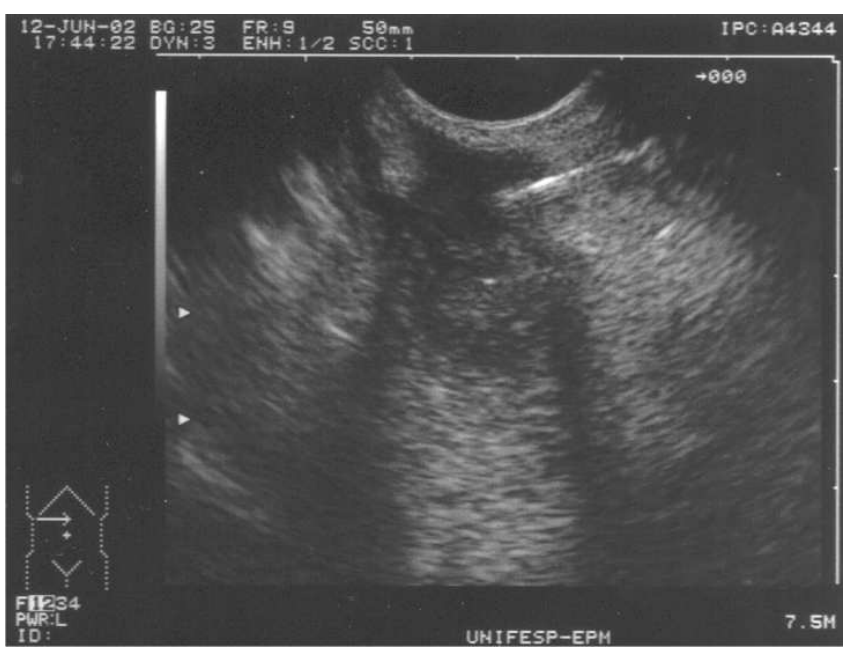

Figura 2 - Momento da EE-PAAF em massa da cabeça do pancreas. 

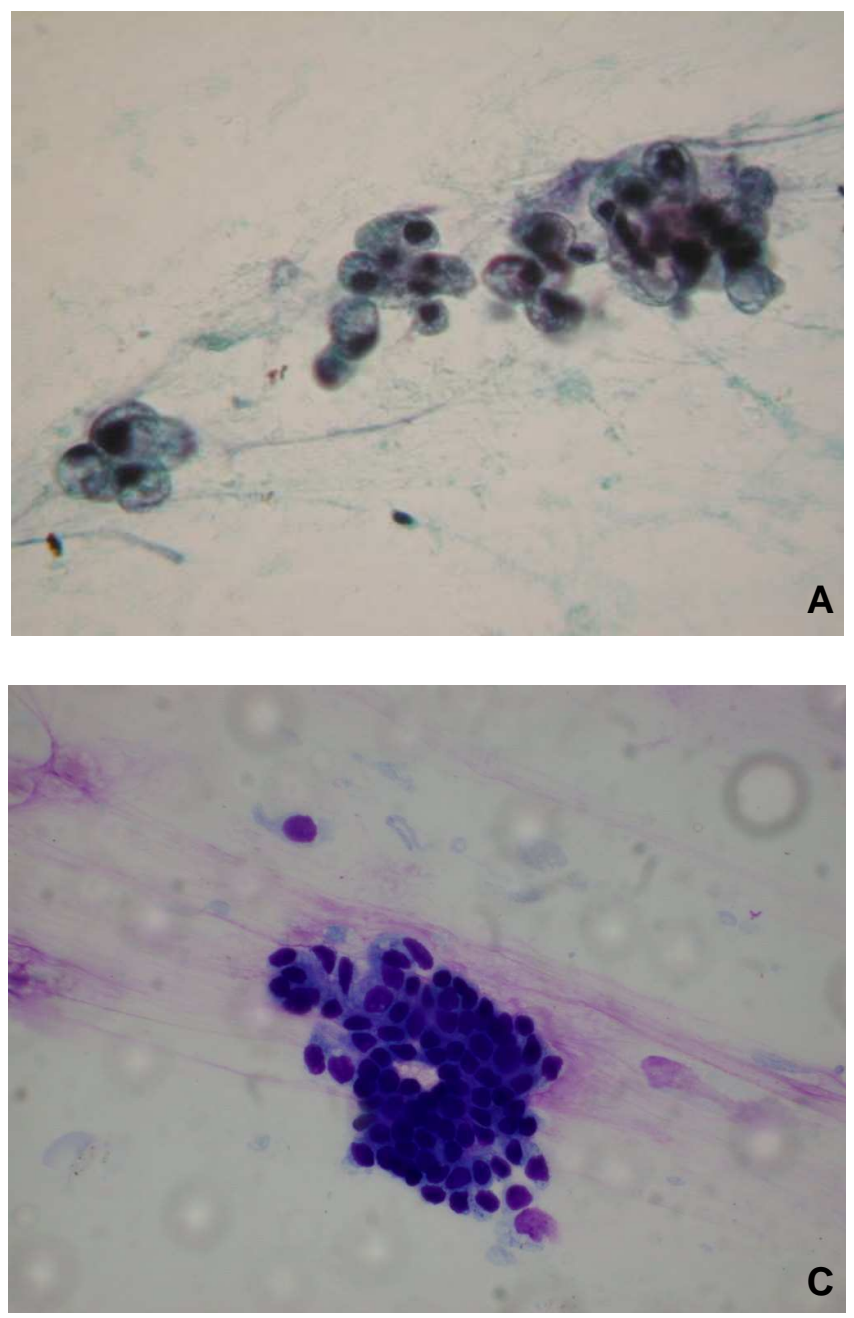

Figura 3 negativa para neoplasia. expressou a possibilidade do diagnóstico de malignidade em um teste citológico positivo. Valores de $p=0,05$ foram considerados significativos. A análise estatística foi realizada utilizando SAS ${ }^{\circledR}$ System 6.04 e SPSS 10.0.

\section{RESULTADOS}

As características iniciais dos pacientes incluídos podem ser apreciados na tabela 1. Vinte e cinco eram do sexo feminino (54\%) com média de idade de 56 anos (4087 anos). A icterícia foi o sintoma de apresentação em 41 casos (89\%). Os demais sintomas incluem: dor abdominal em 34 e perda de peso em 39 pacientes. O seguimento médio foi de 15 meses (média de cinco meses).

A esfincterotomia endoscópica foi realizada em 19 casos para facilitar a colocação subseqüente de próteses. Em 40 pacientes foram colocadas próteses plásticas biliares e em um foi colocada uma prótese plástica pancreática. A pancreatografia foi realizada em 23 casos, especialmente quando havia suspeita de doença pancreática. A EE-PAAF
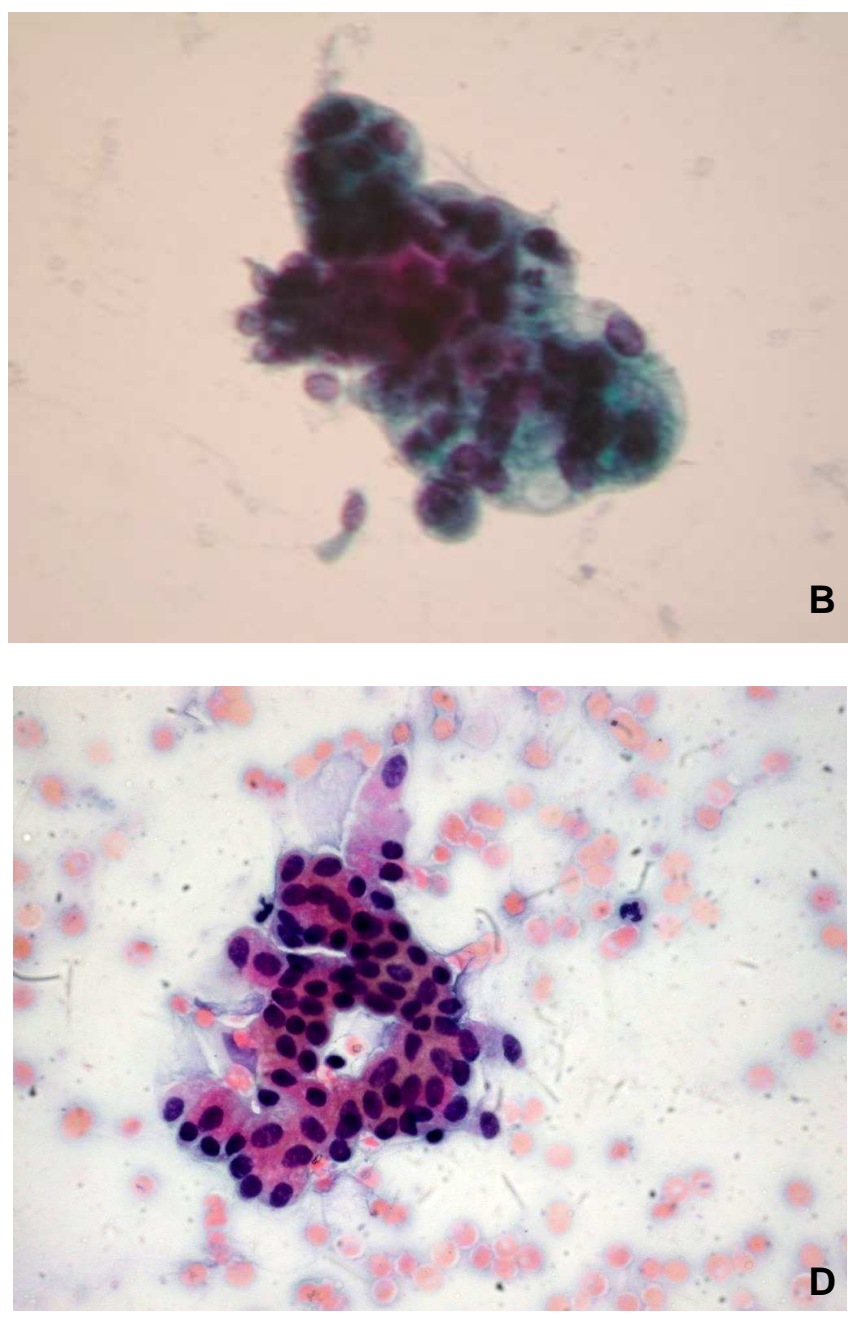

ăo foi realizada em 5 pacientes porque não havia nenhuma lesão tipo massa ou espessamento focal da parede do ducto biliar. Houve cinco complicações da CPER (11\%) e duas da EE-PAAF (5\%). A pancreatite pós CPER foi leve, necessitando apenas de tratamento conservador. Dois pacientes desenvolveram colangite devido à drenagem incompleta e receberam tratamento endoscópico. A obstrução e migração do stent também foi tratada por endoscopia. O sangramento da massa pancreática após EE-PAAF foi leve e autolimitado. Um paciente desenvolveu peritonite biliar após EE-PAAF da massa biliar com perfuração inadvertida da vesícula biliar e foi submetido à laparotomia. Veio a óbito 12 horas após o procedimento. A tabela 2 mostra os procedimentos terapêuticos e as complicações. Dos 37 doentes considerados como portadores de estenose maligna, o diagnóstico histológico foi confirmado em 32: 1 autópsia, 10 cirurgias e 21 citológica e/ou histológica. As malignidades foram ainda separadas em pancreáticas e biliares de acordo com a histopatologia ou evidência clínica, incluindo os achados de CPER, tomografia computadorizada e EE (Tabela 3). 
Tabela 1 - Análise comparativa entre as características clínicas e o diagnóstico final.

\begin{tabular}{|c|c|c|c|c|}
\hline Características Clínicas & Todos os pacientes & Estenoses malignas & Estenoses benignas & $p$ valor \\
\hline Número de Pacientes & 46 & 37 & 9 & - \\
\hline Sexo, n (\%) & & & & $<0,005^{*}$ \\
\hline Masculino & $21 \quad(46)$ & $12(32)$ & $8(89)$ & - \\
\hline Feminino & $25 \quad(54)$ & $25(68)$ & $1(11)$ & - \\
\hline Icterícia, n (\%) & 41 (89) & $36(97)$ & $5(56)$ & $<0,005^{*}$ \\
\hline Perda de peso, n (\%) & 39 (85) & $34(92)$ & $5(56)$ & $<0,02 *$ \\
\hline Dor abdominal, n (\%) & 34 (74) & $26(70)$ & $8(89)$ & $N S^{*}$ \\
\hline Média de Idade (SD) & $56(11)$ & $62(14)$ & $49 \quad(8)$ & $<0,01 \#$ \\
\hline
\end{tabular}

* Teste exato de Fisher \# Test T de Students.

Tabela 2 - Procedimentos endoscópicos terapêuticos e complicações.

\begin{tabular}{lrrr}
\hline Procedimentos terapêuticos & $\mathrm{n}(\%)$ & \\
\hline Esfincterotomia & 19 & $(41)$ & \\
Prótese biliar & 40 & $(87)$ & \\
Prótese pancreática & 1 & $(2)$ & Descrição \\
Complicações & 5 & $(11)$ & $\begin{array}{l}\text { Colangite (2); obstrução da prótese (1); pancreatite (1); } \\
\text { Complicações da CPER }\end{array}$ \\
$\begin{array}{llll}\text { Complicações da da EE } & 2 & (5) & \text { Peritese (1) }\end{array}$ \\
\hline
\end{tabular}

Tabela 3 - Localização e etiologia das estenoses em 46 pacientes.

\begin{tabular}{lrc}
\hline Tumores Malignos $(\mathbf{n}=\mathbf{3 7})$ & N & $(\%)$ \\
\hline Pancreáticos & 26 & $(70)$ \\
Biliares & 11 & $(30)$ \\
DBC & 8 & $(22)$ \\
Hilares & 3 & $(8)$ \\
Condições benigna $(\mathbf{n}=9)$ & & \\
Pancreatite crônica & 8 & $(89)$ \\
Estenose inflamatória do DBC & 1 & $(11)$ \\
\hline
\end{tabular}

DBC: Ducto biliar comum

O desempenho diagnóstico da citologia por escovado colhido por CPER, EE-PAAF e a combinação de ambos os métodos são apresentados nas tabelas 4 e 5 . A acurácia diagnóstica da EE-PAAF foi maior para o patologista especialista GI, quando comparada ao patologista geral. Em comparação a citologia por escovado e EE-PAAF demonstrou maior sensibilidade e acurácia tanto para o patologista geral $(64,9 \%$ e $69,6 \%)$ como para o patologista especialista GI (83,8\% e $84,8 \%)$. A probabilidade de diagnosticar a doença maligna foi de 5,84 para 7,54 vezes, respectivamente, para o patologista geral e especialista.

A concordância geral entre o patologista geral e o especialista foi boa para todos os métodos, com maior coeficiente kappa para a citologia por CPER $(k=0,775)$ (Tabela 6). Como mostrado nas tabelas 7 e 8 , a concordância entre o diagnóstico final e a análise do patologista geral e do especialista foi exata quando um único método foi utilizado. A combinação dos métodos melhorou a concordância do diagnóstico final de ambos os patologistas. 0 coeficiente Kappa foi considerado bom para o patologista especialista. Quando os resultados dos tumores de pâncreas e de vias biliares foram analisados separadamente (Tabela 9) não houve diferença entre a sensibilidade da citologia por CPER e a EE-PAAF.

\section{DISCUSSÃO}

Embora as estenoses biliares sejam comumente encontradas durante a CPER, o diagnóstico confiável permanece um desafio. A melhora do diagnóstico pré-operatório e do estádio é fundamental para aqueles pacientes que serão beneficiados pela ressecção cirúrgica. O diagnóstico histológico é necessário para aqueles que não são candidatos à cirurgia e antes do início da quimioterapia e / ou radioterapia ${ }^{17}$. Tradicionalmente, os pacientes com estenose biliar são avaliados e tratados primeiramente pela $\mathrm{CPER}^{12}$. Assim, a obtenção de espécimes de tais lesões durante este procedimento é o método mais prático para o diagnóstico histológico ${ }^{3}$. A citologia obtida pela CPER é provavelmente a técnica mais usada para colher amostra tecidual, por ser conveniente, rápida e de fácil execução ${ }^{12}$, 
Tabela 4 - Desempenho diagnóstico do escovado obtido pela CPER, da EE-PAAF e por ambos os métodos combinados de acordo com a análise de patologistas gerais.

\begin{tabular}{lcccccc}
\hline Método & Sens. (\%) & Espec. (\%) & VPP (\%) & VPN (\%) & Acurácia (\%) & RV+ \\
\hline Citologia por CPER & 43,2 & 88,9 & 94,1 & 27,6 & 52,2 & 3,89 \\
EE-PAAF & 52,8 & 100,0 & 100,0 & 22,7 & 58,5 & $*$ \\
CPER + EE-PAAF & 64,9 & 88,9 & 96,0 & 38,1 & 69,6 & 5,84 \\
\hline
\end{tabular}

$V P P=$ valor preditivo positivo; $V P N=$ valor preditivo negativo; $R V=$ razão de verossimilhança. *indeterminada.

Tabela 5 - Desempenho diagnóstico do escovado obtido por CPER, da EE-PAAF e por ambos os métodos combinados de acordo com a análise de patologistas gastrointestinais.

\begin{tabular}{lcccccc}
\hline Método & Sens. (\%) & Espec. (\%) & VPP (\%) & VPN (\%) & Acurácia (\%) & RV+ \\
\hline Citologia por CPER & 51,4 & 88,9 & 95,0 & 30,8 & 58,7 & 4,62 \\
EE-PAAF & 69,4 & 100,0 & 100,0 & 31,3 & 73,2 & $*$ \\
CPER + EE-PAAF & 83,8 & 88,9 & 96,9 & 57,1 & 84,8 & 7,54 \\
\hline
\end{tabular}

$V P P=$ valor preditivo positivo; $V P N=$ valor preditivo negativo; $R V=$ razão de verossimilhança. *indeterminada.

Tabela 6 - Concordância global entre patologistas gerais e gastrointestinais.

\begin{tabular}{llccc}
\hline Método & N & Concordância (\%) & Kappa $(\mathrm{SE}) *$ & $p$ \\
\hline Citologia por CPER & 46 & 89,1 & $0,775(0,146)$ & $<0,0001$ \\
EEE-PAAF & 41 & 85,4 & $0,712(0,150)$ & $<0,0001$ \\
CPER + EE-PAAF & 46 & 84,8 & $0,685(0,140)$ & $<0,00001$ \\
\hline
\end{tabular}

* Erro padrão.

Tabela 7 - Concordância global entre patologistas gerais e diagnóstico final.

\begin{tabular}{llccc}
\hline Método & $\mathrm{N}$ & Concordância $(\%)$ & Kappa (SE)* & $p$ \\
\hline Citologia por CPER & 46 & 52,2 & $0,175(0,097)$ & 0,037 \\
EE-PAAF & 41 & 58,5 & $0,214(0,097)$ & 0,013 \\
CPER + EE-PAAF & 46 & 69,6 & $0,357(0,123)$ & 0,002 \\
\hline
\end{tabular}

* Erro padrão.

mas sua sensibilidade nas estenoses biliares malignas continua longe do ideal. Nossos resultados quanto à sensibilidade da citologia pelo escovado foi de 43,2\% e 51,4\%, respectivamente, para o patologista geral e especialista, semelhantes aos publicados anteriormente. Uma análise de 17 estudos encontrou sensibilidade global de $49 \%{ }^{2,18,19}$.

A EE permite obter excelentes imagens do ducto biliar e é usada para detectar e auxiliar no diagnóstico diferencial das massas e estenoses ductais ${ }^{12,20,21}$. Além disso, a EE fornece informação sobre o estádio que não pode ser feito durante a CPER ${ }^{1220,22}$. Estudos prévios determinaram o papel da EE-PAAF na abordagem da estenose biliar. A sensibilidade da EE-PAAF varia de $43 \%$ a $86 \%$ em todas as estenoses biliares e de $25 \%$ a $83 \%$ nas estenoses biliares proximais ${ }^{8-12,17}$.No presente estudo, os resultados da EE-
PAAF para o diagnóstico histológico das estenoses biliares distais foram: sensibilidade de $52,8 \%$ e $69,4 \%$, especificidade de $100 \%$ e acurácia de 58,5\% e 73,2\% para o patologista geral e o especialista $\mathrm{Gl}$, respectivamente. Esses resultados são ligeiramente melhores do que os relatados por Rösch et al. ${ }^{2}$ Lee et al. ${ }^{8}$ encontraram sensibilidade de $47 \%$ para a EE-PAAF no diagnóstico das estenoses biliares em 40 pacientes.

Vários outros estudos incluindo pacientes com estenoses biliares em sua maioria proximais e com citologia anterior obtida pela CPER negativa, demonstraram melhores resultados com a EE-PAAF com sensibilidade que variou de $77 \%$ a $89 \%$, acurácia de $79 \%$ a $91 \%$ e especificidade de 100\% ${ }^{9,11,13}$. As razões para estas diferenças não são claras, já que as lesões hilares são geralmente 
Tabela 8 - Concordância entre patologistas gastrointestinais e diagnóstico final.

\begin{tabular}{lcccc}
\hline CPER (citologia) & $\mathrm{N}$ & Concordância $(\%)$ & Kappa $(\mathrm{SE}) *$ & $p$ \\
\hline EE-PAAF & 46 & 58,7 & $0,235(0,107)$ & 0,014 \\
CPER + EE-PAAF & 41 & 73,2 & $0,357(0,120)$ & 0,001 \\
CPER (citologia) & 46 & 84,8 & $0,600(0,141)$ & $<0,0001$ \\
\hline
\end{tabular}

* Erro padrão.

Tabela 9 - Sensibilidade de ambos os métodos agrupados em tumores pancreáticos e biliares.

\begin{tabular}{|c|c|c|c|}
\hline & Tumores pancreáticos $\mathrm{n}(\%)$ & Tumores biliares $\mathrm{n}(\%)$ & $p$ \\
\hline \multicolumn{4}{|c|}{ Patologistas gerais (PG) } \\
\hline Citologia por CPER & $13 / 26 \quad(50)$ & $3 / 11 \quad(27)$ & 0,18 \\
\hline EE-PAAF & $14 / 25 \quad(56)$ & $5 / 11 \quad(46)$ & 0,55 \\
\hline \multicolumn{4}{|l|}{ Patologistas GI } \\
\hline Citologia por CPER & $15 / 26 \quad(58)$ & $4 / 11 \quad(36)$ & 0,23 \\
\hline EE-PAAF & $18 / 25 \quad(72)$ & $7 / 11 \quad(64)$ & 0,44 \\
\hline
\end{tabular}

difíceis de detectar como também existe dificuldade na coleta de material para citologia, em comparação com as lesões biliares distais. Isto pode ser explicado pelo fato de que o ultrasonografista não tinha conhecimento do resultado citológico anterior. Além disso, a presença de prótese biliar in loco tornou mais fácil a identificação do ducto biliar e localização do tumor em todos os casos. São necessários mais estudos para esclarecer essas diferenças.

Rosch et al. ${ }^{2}$ compararam os testes isolados e obtiveram os melhores resultados com a combinação de CPER e EE-PAAF (sensibilidade $71 \%$, especificidade de $100 \%$ e acurácia $86 \%$ ). No presente trabalho, observamos que a combinação de escovado e EE-PAAF mostrou maior sensibilidade e acurácia tanto para o patologista geral $(64,9 \%$ e $69,6 \%)$ como para o especialista (83,8\% e $84,8 \%$ ), quando comparado aos testes isolados. A probabilidade de doença maligna aumentou de 5,84 para 7,54 vezes, respectivamente, para o patologista geral e especialista em GI. Houve cinco complicações da CPER $(11 \%)$, nenhuma delas diretamente relacionado ao escovado. A taxa de complicação da EE-PAAF neste estudo foi de $5 \%$, que é semelhante a relatada anteriormente $(1 \% \text { a } 5 \%)^{23-26}$.

A concordância global entre o patologista geral e o especialista GI foi boa para os dois métodos. Quando eles discordaram, o patologista GI provou ser mais preciso principalmente em citologia pela EE-PAAF. A concordância diagnóstica final entre os dois patologistas foi melhorada pela combinação dos métodos. Isso foi ainda mais significativo para o patologista Gl, para quem o coeficiente kappa foi considerado bom. A interpretação dos espécimes citológicos é em parte subjetiva e a discordância interobservador é comum ${ }^{27-29}$. Os erros de diagnóstico po- dem estar relacionados à inexperiência do citopatologista. Portanto, o treinamento adequado e o uso de critérios citológicos estabelecidos são obrigatórios ${ }^{1,30}$.

É necessário a compreensão da filosofia de citologistas e patologistas responsáveis pela interpretação de esfregaços citológicos. Um citopatologista "agressivo" diagnostica malignidade com base na presença de poucas alterações celulares características, resultando em alta sensibilidade, porém com especificidade mais baixa (mais resultados falso-positivos) $)^{18}$. Por outro lado, um citopatologista "conservador", pode hesitar em rotular um espécime como maligno, exigindo alterações definidas (óbvias) antes de fazer esse diagnóstico, resultando em menor sensibilidade, porém com maior especificidade (poucos resultados falso-positivos) ${ }^{31}$. Espera-se que aqueles que se dedicam totalmente à patologia Gl interpretem as amostras com maior precisão do que os patologistas gerais que avaliam amostras de diferentes órgãos ${ }^{1,32}$.

Quando apenas os escovados positivos ou suspeitos de malignidade foram considerados equivalentes ao câncer (a citologia atípica foi considerada como benigna), observou-se um aumento da especificidade (100\%) e uma ligeira redução da sensibilidade e acurácia ao combinar os métodos tanto para o patologista geral como para o especialista Gl. Nesta situação, o escovado, a EUS-FNA e a combinação de ambos diminuiu a sensibilidade para $46 \%$, $66,7 \%$ e $78,4 \%$, respectivamente em relação ao patologista especialista $\mathrm{Gl}$.

É possível que o desenvolvimento de novos instrumentos como agulhas para core biópsia possa aumentar a sensibilidade da EE-PAAF ${ }^{33}$. Relata-se que o Doppler com contraste pode diferenciar as massas inflamatórias e malignas do pâncreas ${ }^{34}$, mas se isso melhora o desempe- 
nho das biópsias obtidas pela EE-PAAF ainda não foi determinado².

A taxa de detecção de câncer de todas as técnicas é influenciada pelo tipo de tumor responsável pela estenose biliar. Na maioria das séries, a citologia do escovado e do material obtido com pinça de biópsia tem maior sensibilidade para o colangiocarcinoma (44\% a $100 \%$ ) do que para o câncer do pâncreas $(30 \% \text { a } 65 \%)^{3}$. Rösch et al. ${ }^{2}$ concluíram que os resultados do escovado obtido pela CPER parece ser superior na estenose biliar, enquanto a EE-PAAF parece ser melhor nas massas pancreáticas. No entanto, essas diferenças não foram significativas. No presente estudo não houve diferença significativa quanto à sensibilidade do escovado obtido por CPER e a EE-PAAF no diagnóstico citológico dos tumores pancreáticos e biliares. Não existe qualquer comparação direta entre a EE-PAAF no diagnóstico da neoplasia pancreática versus biliar, embora uma série sugira que a acurácia da EE-PAAF para o câncer do ducto biliar seja boa ${ }^{10}$.

Nosso estudo apresenta déficits potenciais. Primeiro, a amostra pode não ser grande o suficiente. No entanto, este é um dos maiores estudos que compara a CPER a EE-PAAF na coleta de amostra tecidual de estenoses biliares. Segundo, a nossa população de doentes não pode ser semelhante à observada em instituições de atendimento primário. No entanto, atualmente deve ser conduta padrão encaminhá-los às instituições especializadas. Por outro lado, nosso estudo apresenta algumas vantagens em relação a experiências anteriores. Em primeiro lugar, nosso estudo foi prospectivo. Segundo foi utilizada uma metodologia protocolar rigorosa. Em terceiro lugar, nossos resultados têm implicações clínicas potenciais, pois mostramos que a CPER e a EE-PAAF têm resultados semelhantes. Além disso, se ambas as técnicas forem usadas de forma combinada a acurácia diagnóstica aumenta. E finalmente, avaliamos a acurácia diagnóstica do patologista regular versus especialista. É do nosso conhecimento que esta questão não foi abordada anteriormente em nenhum estudo nacional ou internacional. Acreditamos realmente que este aspecto é também de importância clínica.

Em resumo, este estudo prospectivo mostra que embora tanto a citologia por CPER quanto as amostras teciduais obtidas pela EE-PAAF apresentem limitações quanto ao diagnóstico diferencial das estenoses biliares, a acurácia diagnóstica é aceitável, e a combinação de métodos resulta em um maior desempenho. Além disso, espera-se que os patologistas especialistas Gl interpretem as amostras com maior acurácia do que os patologistas gerais.

\section{A B S S T R A C}

Objective: To evaluate and to compare the diagnostic yield of ERCP brush cytology (ERCP) and EUS-FNA in patients with biliary strictures and evaluates the agreement between general pathologists (GP) and expert Gl pathologists (GIP) in the final diagnosis of biliary strictures. Methods: Patients with biliary strictures documented by ERCP were included. Brush cytology was performed and during EUS, only visible mass lesions or localized bile duct wall thickening were aspirated. The gold standard method for diagnosis was surgical histology and/or follow-up. Tissue sampling results were: malignant, suspicious, atypical, insufficiently or benign. Specimens were interpreted by GP and GIP, blinded for prior tests results. Results: 46 patients were included. Final diagnosis was malignancy in 37 (26 pancreatic - 11 biliary) and benign in 9 (8 chronic pancreatitis - 1 common bile duct inflammatory stricture). Sensitivity and accuracy for ERCP brush cytology were $43.2 \%$ and $52.2 \%$ for GP and $51.4 \%$ and $58.7 \%$ for GIP. Sensitivity and accuracy for EUS-FNA were $52.8 \%$ and $58.5 \%$, respectively for GP and $69.4 \%$ e $73.2 \%$ for GIP. In comparison, the combination of brush cytology and EUS-FNA demonstrated higher sensitivity and accuracy for both GP $(64.9 \%$ and $69.6 \%$, respectively) and GIP (83.8\% and $84.8 \%$, respectively) and improved agreement with final diagnosis for both (mostly for GIP). Conclusion: Both, ERCP brush cytology and EUS-FNA has a similar yield for the diagnosis of biliary strictures. However, the combination of these methods results in an improved diagnostic accuracy. In addition, GIP might be expected to interpret specimens with greater accuracy than GP.

Keywords: Bile ducts/cytology. Biliary tract neoplasms/pathology. Cholangiopancreatography, endoscopic retrograde. Cholestasis, extrahepatic/pathology/ultrasonography. Endosonography/methods.

\section{REFERENCIAS}

1. De Bellis $M$, Sherman $S$, Fogel EL, Cramer $H$, Chappo J, McHenry $L J r$ et al. Tissue sampling at ERCP in suspected malignant biliary strictures (Part 1). Gastrointest Endosc. 2002; 56(4):552-61.

2. Rösch $T$, Hofrichter $K$, Frimberger $E$, Meining $A$, Born $P$, Weigert $N$ et al. ERCP or EUS for tissue diagnosis of biliary strictures? A prospective comparative study. Gastrointest Endosc. 2004; 60(3):390-6.
3. Farrell RJ, Jain AK, Brandwein $\mathrm{SL}$, Wang $\mathrm{H}$, Chuttani R, Pleskow DK. The combination of stricture dilation, endoscopic needle aspiration, and biliary brushings significantly improves diagnostic yield from malignant bile duct strictures. Gastrointest Endosc. 2001; 54(5):587-94.

4. Wamsteker EJ. Updates in biliary endoscopy. Curr Opin Gastroenterol. 2006; 22(3):300-4.

5. De Bellis M, Sherman S, Fogel EL, Cramer H, Chappo J, McHenry $\mathrm{L} \mathrm{Jr}$ et al. Tissue sampling at ERCP in suspected malignant biliary strictures (Part 2). Gastrointest Endosc. 2002; 56(5):720-30. 
6. Lee JG, Leung J. Tissue sampling at ERCP in suspected pancreatic cancer. Gastrointest Endosc Clin N Am. 1998; 8(1):221-35.

7. Fogel EL, deBellis M, McHenry L, Watkins JL, Chappo J, Cramer H et al. Effectiveness of a new long cytology brush in the evaluation of malignant biliary obstruction: a prospective study. Gastrointest Endosc. 2006; 63(1):71-7.

8. Lee JH, Salem R, Aslanian H, Chacho M, Topazian M. Endoscopic ultrasound and fine-needle aspiration of unexplained bile duct strictures. Am J Gastroenterol. 2004; 99(6):1069-73.

9. Eloubeidi MA, Chen VK, Jhala NC, Eltoum IE, Jhala D, Chhieng DC et al. Endoscopic ultrasound-guided fine needle aspiration biopsy of suspected cholangiocarcinoma. Clin Gastroenterol Hepatol. 2004; 2:(3)209-13.

10. Fritscher-Ravens A, Broering DC, Sriram PV, Topalidis T, Jaeckle $S$, Thonke F, Soehendra N. EUS-guided fine-needle aspiration cytodiagnosis of hilar cholangiocarcinoma: a case series. Gastrointest Endosc. 2000; 52(4):534-40.

11. Fritscher-Ravens A, Broering DC, Knoefel WT, Rogiers $X$, Swain $P$, Thonke $F$ et al. EUS-guided fine-needle aspiration of suspected hilar cholangiocarcinoma in potentially operable patients with negative brush cytology. Am J Gastroenterol. 2004; 99(1):45-51.

12. Byrne MF, Gerke H, Mitchell RM, Stiffler HL, McGrath K, Branch MS et al. Yield of endoscopic ultrasound-guided fine-needle aspiration of bile duct lesions. Endoscopy. 2004; 36(8):715-9.

13. DeWitt J, Misra VL, Leblanc JK, McHenry L, Sherman S. EUSguided FNA of proximal biliary strictures after negative ERCP brush cytology results. Gastrointest Endosc. 2006; 64(3):325-33.

14. World Health Organization classification of tumors. Pathology and genetics. IARC Press; 2000.

15. Kline T. Pancreas. Handbook of fine needle aspiration biopsy cytology. St Louis: Mosby; 1988. p.317-41.

16. Rabinovitz M, Zajko AB, Hassanein $T$, Shetty B, Bron KM, Schade $R R$ et al. Diagnostic value of brush cytology in the diagnosis of bile duct carcinoma: a study in 65 patients with bile duct strictures. Hepatology. 1990; 12(4 Pt 1):747-52.

17. Pavey DA, Gress FG. The role of EUS-guided FNA for the evaluation of biliary strictures. Gastrointest Endosc. 2006; 64(3):334-7.

18. Glasbrenner B, Ardan M, Boeck W, Preclik G, Moller P, Adler G. Prospective evaluation of brush cytology of biliary strictures during endoscopic retrograde cholangiopancreatography. Endoscopy. 1999; 31(9):712-7.

19. Jailwala J, Fogel EL, Sherman S, Gottlieb K, Flueckiger J, Bucksot LG, Lehman GA. Triple-tissue sampling at ERCP in malignant biliary obstruction. Gastrointest Endosc. 2000; 51(4 Pt 1):383-90.

20. Mukai H, Yasuda K, Nakajima M. Tumors of the papilla and distal common bile duct. Diagnosis and staging by endoscopic ultrasonography. Gastrointest Endosc Clin N Am.1995; 5(4):76372 .

21. Ardengh JC, Lopes CV, de Lima LF, de Oliveira JR, Venco F, Santo GC, Modena JL. Diagnosis of pancreatic tumors by endoscopic ultrasound-guided fine-needle aspiration. World J Gastroenterol. 2007; 13(22):3112-6

22. Ardengh JC, Malheiros CA, Pereira V, Coelho DE, Coelho JF, Rahal F. Endoscopic ultrasound-guided fine-needle aspiration using helical computerized tomography for TN staging and vascular injury in operable pancreatic carcinoma. JOP. 2009; 10(3):310-7.

23. Voss M, Hammel P, Molas G, Palazzo L, Dancour A, O'Toole D et al. Value of endoscopic ultrasound guided fine needle aspiration biopsy in the diagnosis of solid pancreatic masses. Gut. 2000; 46(2):244-9.
24. Gress F, Gottlieb K, Sherman S, Lehman G. Endoscopic ultrasonography-guided fine-needle aspiration biopsy of suspected pancreatic cancer. Ann Intern Med. 2001; 134(6):459-64.

25. O'Toole D, Palazzo L, Arotcarena R, Dancour A, Aubert A, Hammel $P$ et al. Assessment of complications of EUS-guided fine-needle aspiration. Gastrointest Endosc. 2001; 53(4):470-4.

26. Eloubeidi MA, Tamhane A, Varadarajulu S, Wilcox CM. Frequency of major complications after EUS-guided FNA of solid pancreatic masses: a prospective evaluation. Gastrointest Endosc. 2006; 63(4):622-9.

27. Hopman EH, Voorhorst FJ, Kenemans P, Meyer CJ, Helmerhorst TJ. Observer agreement on interpreting colposcopic images of CIN. Gynecol Oncol. 1995; 58(2):206-9.

28. Gupta DK, Komaromy-Hiller G, Raab SS, Nath ME. Interobserver and intraobserver variability in the cytologic diagnosis of normal and abnormal metaplastic squamous cells in pap smears. Acta Cytol. 2001; 45(5):697-703.

29. Hahm GK, Niemann TH, Lucas JG, Frankel WL. The value of second opinion in gastrointestinal and liver pathology. Arch Pathol Lab Med. 2001; 125(6):736-9.

30. Cohen MB, Wittchow RJ, Johlin FC, Bottles K, Raab SS. Brush cytology of the extrahepatic biliary tract: comparison of cytologic features of adenocarcinoma and benign biliary strictures. Mod Pathol. 1995; 8(5):498-502.

31. de Peralta-Venturina MN, Wong DK, Purslow MJ, Kini SR. Biliary tract cytology in specimens obtained by direct cholangiographic procedures: a study of 74 cases. Diagn Cytopathol. 1996; 14(4):33448.

32. Rex DK, Alikhan M, Cummings O, Ulbright TM. Accuracy of pathologic interpretation of colorectal polyps by general pathologists in community practice. Gastrointest Endosc. 1999; 50(4):468-74.

33. Larghi A, Verna EC, Stavropoulos SN, Rotterdam H, Lightdale CJ, Stevens PD. EUS-guided trucut needle biopsies in patients with solid pancreatic masses: a prospective study. Gastrointest Endosc. 2004; 59(2):185-90.

34. Becker D, Strobel D, Bernatik T, Hahn EG. Echo-enhanced colorand power-Doppler EUS for the discrimination between focal pancreatitis and pancreatic carcinoma. Gastrointest Endosc. 2001; 53(7):784-9.

Recebido em 17/03/2009

Aceito para publicação em 20/05/2009

Conflito de interesse: nenhum

Fonte de financiamento: nenhuma

\section{Como citar esse artigo:}

Novis M, Ardengh JC, Libera ED, Nakao FS, Ornellas LC, Santo GC, Venco F, Ferrari AP. Estudo prospectivo e comparativo do escovado obtido pela colangiopancreatografia endoscópica retrógrada (CPER) a ecoendoscopia associada à punção aspirativa com agulha fina (EEPAAF) no diagnóstico diferencial das estenoses biliares. Rev Col Bras Cir. [periódico na Internet] 2010; 37(3). Disponível em URL: http:// www.scielo.br/rcbc

\section{Endereço para correspondência:}

José Celso Ardengh

E-mail: jcelso@uol.com.br 